\title{
Progressive Thoracic Kyphoscoliosis leading to Paraplegia in a Child with Neurofibromatosis Type-1
}

\author{
Sumeera Matee ${ }^{1}$, Saeed Bin Ayaz ${ }^{2}$ and Uzma Bashir ${ }^{3}$ \\ ${ }^{1}$ Department of Rehabilitation Medicine, Combined Military Hospital, Mangla, Punjab, Pakistan \\ ${ }^{2}$ Department of Rehabilitation Medicine, Combined Military Hospital, Jhelum, Punjab, Pakistan \\ ${ }^{3}$ Department of Dermatology, Combined Military Hospital, Quetta, and Quetta Institute of Medical Sciences, Quetta, Pakistan
}

\begin{abstract}
In this case report we describe a case of a 13-year male with neurofibromatosis type-1, who had been suffering from progressive thoracic kyphoscoliosis that resulted in spinal cord compression and paraplegia. The report is meant to highlight the importance of timely detection and adequate follow-up of spinal deformities, associated with neurofibromatosis. Such deformities, if not effectively addressed, may progress corresponding to skeletal growth and lead to secondary problems, as was seen in our patient, who ultimately developed paraplegia.
\end{abstract}

Key Words: Neurofibromatosis, Kyphoscoliosis, Paraplegia.

How to cite this article: Matee S, Ayaz SB, Bashir U. Progressive Thoracic Kyphoscoliosis leading to Paraplegia in a Child with Neurofibromatosis Type-1. J Coll Physicians Surg Pak 2021; 31(01):98-100.

\section{INTRODUCTION}

Neurofibromatosis (NF) is an autosomal dominant disorder of neural crest cell origin. ${ }^{1}$ It has two subtypes (type 1 and type 2). NF1 accounts for $90 \%$ of all cases of NF with no sex or racial predilection. ${ }^{1}$ The genetic makeup in NF1 predisposes the patients to develop multiple neurofibromas. Neurofibromas are benign tumors of the endoneurium and are frequently associated with compression of spinal cord, if they arise from spinal nerve roots. ${ }^{2}$

The spine in NF1 may be defective irrespective of compression effects from a neurofibroma. The spinal deformity observed in these individuals may result from gradual scoliotic rotation and progression or it may occur early in the disease with an abrupt angular kyphotic curve. ${ }^{3}$ This scoliotic deformity is categorised into two types: dystrophic and non-dystrophic. Dystrophic scoliosis is manifested by erosions of vertebral body margins, rotation of apical vertebra, rib pencilling, wedging of one or more vertebral bodies, paraspinal or intraspinal soft tissue masses, and dural ectasias. ${ }^{4,5}$ Scoliotic deformity is very rarely associated with neurological paralysis. We report herein, a teenager with NF1, who presented with a giant paraspinal neurofibroma and progressive thoracic dystrophic kyphoscoliosis, ultimately leading to spinal cord compression and paraplegia.

Correspondence to: Dr. Sumeera Matee, Department of Rehabilitation Medicine, Combined Military Hospital Mangla, Punjab, Pakistan

E-mail: sumeera_matee@hotmail.com

Received: December 10, 2018; Revised: January 12, 2020;

Accepted: January 20, 2020

DOI: https://doi.org/10.29271/jcpsp.2021.01.98

\section{CASE REPORT}

A 13-year male presented with complaints of inability to walk with progressive weakness and stiffness of legs for the past one and a half years. There was associated bowel and bladder incontinence. He did not report breathing difficulty, dizziness, and auditory or visual problems. According to his parents, he had a swelling on his upper back since birth. They further reported that a tissue biopsy was taken from the swelling at the age of 4 years. The patient was neither advised any treatment at that time, nor did he have the biopsy report with him. His swelling progressively increased in size and at the age of 7 years, he underwent a neurosurgical consultation. X-rays and magnetic resonance imaging (MRI) of his spine (Figure 1 ) were carried out which showed a severe kyphotic deformity in the mid-dorsal region with wedge collapse of the involved vertebrae. There were mixed intensity signals in the regional paraspinal areas that could be tracked in the prevertebral area as well as along the left psoas muscle. Enhancing pockets of collection were also seen in the region of quadratus lumborum as well as in the left renal bed. Severe degree of cord compression was also appreciated secondary to gibbus deformity.

Based on MRI report, the neurosurgeon suspected him of having tuberculous spondylitis and performed exploratory surgery in an attempt to drain the abscess and fix the spine. However, during surgery, he did not find any abscess and rather found a tumor, the bulk of which was removed during surgery. Histopathological evaluation of the tumor showed hypocellular proliferation of elongated spindle shaped cells with few mast cells. As a precautionary measure, after operation, he was prescribed anti-tuberculosis treatment for 12 months. The tumor again started increasing in size and at the age of $11-1 / 2$ years, the 
patient developed stiffness in legs, incontinence, and difficulty in walking. His symptoms were progressive. By the age of 13 years, he was unable to walk, and became completely dependent in activities of daily living.

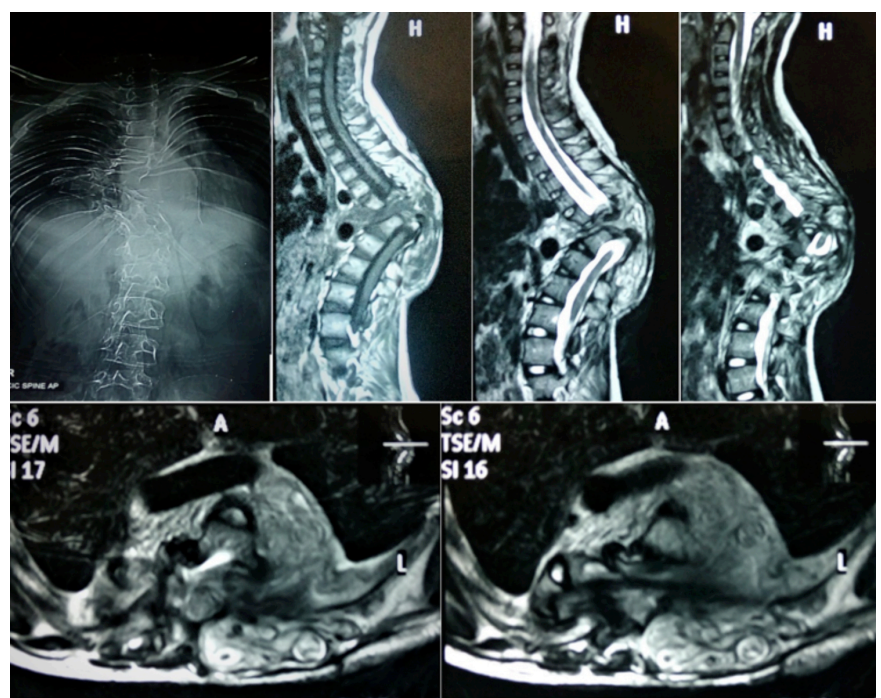

Figure 1: Figure showing severe kyphotic deformity in dorsal spine with collapse of the involved vertebrae along with severe cord compression.

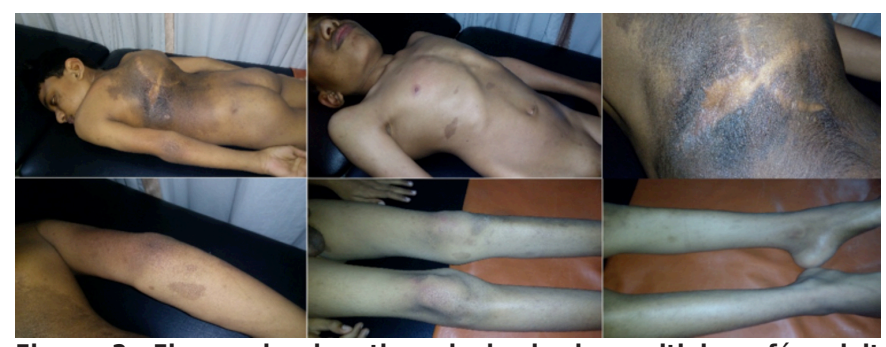

Figure 2: Figure showing thoracic kyphosis, multiple café-au-lait patches, and a hyperpigmented patch over the back with scar marks of previous surgery.

On examination, he had thoracic kyphosis with head size proportionately larger than the trunk. Cutaneous examination revealed a hyperpigmented patch, around $40 \times 50 \mathrm{~cm}$, over the back extending over the thoracolumbar region and crossing the midline (giant neurofibroma). There was increased hair growth and rugosity of the skin over the patch. Two atrophic scar marks of previous surgery were also visible in the patch (Figure-2). Multiple café-au-lait patches (number around 40) varying in size from $0.5-3 \mathrm{~cm}$ were present on limbs, trunk, and face. A further probe into the family history revealed occurrence of similar marks in his brother. Neurological examination revealed a spasticity of grade $2+$ in lower limbs, according to the modified Ashworth scale. Power was 0/5 in legs, according to medical research council scale. ${ }^{6}$ The deep tendon reflexes were grade $3+$ in knees and grade 4+ in ankles, bilaterally. Sensations were intact in legs including anal sensations, but anal tone was absent. Plantars were upgoing bilaterally. Rest of the systemic examinations, including ocular examination and examination of his oral cavity, were unremarkable. Based on the above findings, a diagnosis of spinal cord compression at the level of L1 with American spinal injury association impairment score (AIS) ${ }^{7}$
B was made. The laboratory evaluation revealed normal values for complete blood count, C-reactive protein, erythrocyte sedimentation rate, parathormone, calcium, phosphate, liver and renal function tests, and urine routine examination.

He was advised surgical correction of his deformity by the neurosurgeon, which he refused. He also refused any sort of bracing for legs. Thereupon, he was instructed exercises including stretching of hamstrings, gastrocnemius, soleus, and hip adductor muscles. To control spasticity, baclofen $10 \mathrm{mg}$ thrice a day was advised. The child is under training for wheel chair mobility, transfer techniques, and bowel/bladdercare.

\section{DISCUSSION}

NF1 is associated with spinal deformities that may result from gradual scoliotic rotation and progression or it occur early in the disease characterised by an abrupt angular kyphotic curve.

The central nervous system manifestations affect $15 \%$ of children with $\mathrm{NF1}{ }^{8}$ When a neurological deficit is present, it is usually caused by increasing deformity, pressure effects of an intraspinal or paraspinal tumor, penetration of the ribs into the spinal canal, structural instability of the vertebral column, fibrofatty tissue reaction or dural ectasia. ${ }^{4}$

We reviewed reports of 20 cases with spinal deformity, producing neurological deficits. The mean age of the patients was $22.2 \pm 14.5$ years (range: 8-54 years). Among these, 15 (75\%) were males while five $(25 \%)$ were females. Out of these, $10(50 \%)$ had a cervical problems, while $10(50 \%)$ had a thoracic level of primary spinal deformity. A coexisting large neurofibroma was present in seven (35\%) cases. Surgery was refused by one patient; whereas, remaining 19 underwent a surgical procedure. In one case, the outcome was not mentioned. Sixteen out of 18 cases (88.9\%) had improvement in their neurological outcome; while, two did not observe any change. Halo-traction was attempted in one patient without subsequent surgery which resulted in aggravation of neurological deficit. However, in four cases where halo-traction was followed by surgery, there was complete recovery in three cases, while one had a partial motor recovery.

The dystrophic spinal deformities in NF1 should be treated promptly, as there is a strong tendency for curve progression even following spinal fusion. ${ }^{4}$ Attempts for correction through bracing are mostly ineffective and early aggressive surgical intervention is the acceptable treatment. ${ }^{4,9}$ Curves less than 20 o can be closely observed at 6 -month interval until there is a rapid progression that would prompt surgical management. ${ }^{4}$ Except for patients with scoliotic deformities measuring 20-400 with less than 500 of kyphosis, combined anterior and posterior spinal fusion is the most effective surgical approach. ${ }^{4,10}$ The primary goal of surgery is to remove compression on the neurological elements and stabilise the vertebral column to halt further progression of the deformity. ${ }^{4}$ Total correction of deformity sometimes may potentially aggravate the neurological deficit. $^{4}$ 
In conclusion, spinal deformity in patients with NF1 may cause significant morbidity. A holistic approach towards diagnosis of dystrophic changes on plain radiography and MRI is needed to establish prognosis and management choices. Dystrophic scoliotic curvatures or multiplanar spinal deformities with significant sagittal decompensation necessitate early aggressive surgical management. This report highlights the importance of early aggressive treatment of dystrophic kyphoscoliosis in a child with NF1. A timely surgical management could have protected this child from permanent disability.

\section{PATIENT'S CONSENT:}

The informed consent was obtained from the father of the patient to publish the data concerning the patient.

\section{CONFLICT OF INTEREST:}

The authors declared no conflict of interest.

\section{AUTHORS' CONTRIBUTION:}

SM: Manuscript writing, literature search, revision.

SBA: Patient evaluation, information collection, manuscript writing, literature search.

UB: Patient evaluation, information collection, drafting the article.

\section{REFERENCES}

1. Ghalayani P, Saberi Z, Sardari F. Neurofibromatosis type I (von Recklinghausen's disease): A family case report and literature review. Dent Res J (Isfahan) 2012; 9(4):483-8.

2. Radtke HB, Sebold CD, Allison C, Haidle JL, Schneider G. Neurofibromatosis type 1 in genetic counseling practice: Recommendations of the national society of genetic counselors. J Genet Couns 2007; 16(4):387-407. doi: 10.1007/ s10897-007-9101-8.

3. Crawford AH, Herrera-Soto J. Scoliosis associated with neurofibromatosis. Orthop Clin North Am 2007; 38(4): 553-62, vii. doi: 10.1016/j.ocl.2007.03.008.

4. Tsirikos Al, Saifuddin A, Noordeen MH. Spinal deformity in neurofibromatosis type-1: diagnosis and treatment. Eur Spine J 2005; 14(5):427-39. doi: 10.1007/s00586-0040829-7.

5. Kurosawa T, Yurube T, Kakutani K, Maeno K, Uno K, Kurosaka $M$, et al. Thoracic compression myelopathy due to the progression of dystrophic scoliosis, the presence of a paraspinal tumor, and high and excessive amplitude movement of the shoulder. J Orthop Surg (Hong Kong) 2017; 25(1):2309499016684726. doi: 10.1177/23094990 16684726.

6. Compston A. Aids to the investigation of peripheral nerve injuries. Medical Research Council: Nerve Injuries Research Committee. His Majesty's Stationery Office: 1942; pp. 48 (iii) and 74 figures and 7 diagrams; with aids to the examination of the peripheral nervous system. By Michael O'Brien for the Guarantors of Brain. Saunders Elsevier 2010; pp. [8] 64 and 94 Figures. Brain 2010; 133(10): 2838-44. doi: 10.1093/brain/awq270.

7. Schuld C, Franz S, Brüggemann K, Heutehaus L, Weidner N, Kirshblum SC, et al. International standards for neurological classification of spinal cord injury: impact of the revised worksheet (revision 02/13) on classification performance. J Spinal Cord Med 2016; 39(5):504-12. doi: 10.1080/ 10790268.2016.1180831.

8. Kaufmann D, Müller R, Bartelt B, Wolf M, Kunzi-Rapp K, Hanemann CO, et al. Spinal neurofibromatosis without café-au-lait macules in two families with null mutations of the NF1 gene. Am J Hum Genet 2001; 69(6):1395-400. doi: $10.1086 / 324648$.

9. Zhao X, Li J, Shi L, Yang L, Wu ZX, Zhang DW, et al. Surgical treatment of dystrophic spinal curves caused by neurofibromatosis type 1: A retrospective study of 26 patients. Medicine (Baltimore) 2016; 95(14): e3292. doi: 10.1097/ MD.0000000000003292.

10. Hossein-Pourfeizi H, Tabrizi A, Ganjpours-Sales J, Kiani MR, Shams-Vahadat S. Evaluation surgical treatment results of scoliosis in patients with neurofibromatosis. J Anal Res Clin Med 2014; 2(3): 128-34. doi: 10.5681/jarcm.2014.022 